\title{
Spiders (Aranei) of the Novaya Zemlya Archipelago and the Vaygach Island, Russia
}

\section{Пауки (Aranei) архипекага Новая Земля и о-ва Вайгач, Россия}

\author{
A.V. Tanasevitch \\ A.B. Танасевич
}

\begin{abstract}
A.N. Severtsov Institute of Ecology and Evolution, Russian Academy of Sciences, Leninsky prospekt, 33, Moscow 119071, Russia. E-mail: tanasevitch@gmail.com

Институт проблем экологии и эволюции им. А.Н. Северцова РАН, Ленинский проспект, 33, Москва 119071, Россия.

KEY WORDS: Arctic archipelagoes, islands, polar deserts, arctic tundra, new records.

КЛЮЧЕВЫЕ СЛОВА: Арктические архипелаги, острова, полярные пустыни, арктические тундры, новые находки.
\end{abstract}

ABSTRACT. The spider fauna of the Novaya Zemlya Archipelago and the Vaygach Island is reviewed, with some misidentifications corrected, and the geographical coordinates and modern locality names adjusted. The record of Agyneta rurestris (C.L. Koch, 1836) [M. Dahl, 1928] from the Novaya Zemlya actually refers to $A$. similis (Kulczyński, 1926). The record of Erigone longipalpis (Sundevall, 1830) from the archipelago [M. Dahl, 1928] is based on misidentifying the males of $E$. longipalpis which actually represent $E$. arctica palaearctica Braendegaard, 1934, and the females which in fact belong to E. remota L. Koch, 1869. Macrargus solitarius M. Dahl, 1928, previously considered as a junior synonym of Masikia indistincta (Kulczyński, 1908) [Eskov, Marusik, 1994], is actually a junior synonym of E. psychrophila Thorell, 1872 (syn.n.). New data on the spiders of Novaya Zemlya and Vaygach are presented, based on fresh material. Agyneta similis, Arcterigone pilifrons (L. Koch, 1879), E. arcticola Chamberlin et Ivie, 1947, Gibothorax tchernovi Eskov, 1989, Hilaira nivalis Holm, 1937, H. proletaria (L. Koch, 1879) and M. indistincta are recorded from Novaya Zemlya for the first time. Bathyphantes gracilis (Blackwall, 1841), Mecynargus sphagnicola (Holm, 1939), Semljicola alticola (Holm, 1950) and Silometopoides sphagnicola Eskov et Marusik, 1992 are new to the spider fauna of Vaygach. Erigone arctophylacis Crosby et Bishop, 1928 is to be ejected from the Russian spider list as its single record from the Russian territory (Novaya Zemlya) is a mistake and actually refers to E. arcticola. Considering the above misidentifications and new data, the spider faunas of the Novaya Zemlya Archipelago and the Vaygach Island are currently known to contain 21 and 20 species, respectively.

РЕЗЮМЕ. Обзор фауны пауков архипелага Новая Земля и о-ва Вайгач. Исправлены ошибочные определения, уточнены географические координаты и современные названия мест находок. Отмеченный на Новой Земле Agyneta rurestris (C.L. Koch, 1836) [M. Dahl, 1928], в действительности является A. similis (Kulczyński, 1926). Указание Erigone longipalpis (Sundevall, 1830) для архипелага [M. Dahl, 1928] ошибочно, причём самцы, определённые как E. longipalpis относятся к E. arctica palaearctica Braendegaard, 1934, самки - к E. remota L. Koch, 1869. Macrargus solitarius M. Dahl, 1928, ранее считавшийся младшим синонимом Masikia indistincta (Kulczyński, 1908) [Eskov, Marusik, 1994], в действительности является младшим синонимом $E$. psychrophila Thorell, 1872 (syn.n.). Приведены новые данные по паукам Новой Земли и о-ва Вайгач. Виды A. similis, Arcterigone pilifrons (L. Koch, 1879), E. arcticola Chamberlin et Ivie, 1947, Gibothorax tchernovi Eskov, 1989, Hilaira nivalis Holm, 1937, H. proletaria (L. Koch, 1879) и M. indistincta впервые отмечены для Новой Земли. Виды Bathyphantes gracilis (Blackwall, 1841), Mecynargus sphagnicola (Holm, 1939), Semljicola alticola (Holm, 1950) и Silometopoides sphagnicola Eskov et Marusik, 1992 новые для о-ва Вайгач. Вид E. arctophylacis Crosby et Bishop, 1928 должен быть изъят из списка отечественной фауны пауков, так как единственная его находка на территории России (Новая Земля) в действительности относится к E. arcticola. С учётом ошибочных определений и новых данных, фауна пауков Новой Земли и о-ва Вайгач насчитывает 21 и 20 видов, соответственно.

\section{Introduction}

The first information concerning the spiders of the Novaya Zemlya Archipelago and the Vaygach Island is contained in the works of Ehlers [1873a, b], Heuglin [1874] and L. Koch [1879]. The latter paper was based 
on the abundant material collected during the Swedish expedition to Novaya Zemlya and Yenisei in 1875 , led by A. Nordenskiöld. Only one species was recorded from Novaya Zemlya by Ehlers [1873a, b] and Heuglin [1874], whereas L. Koch's list [1879] contained 19 spider species collected from both Novaya Zemlya and Vaygach. A century later, all material collected by the Nordenskiöld's expedition was revised by Holm [1973], who corrected many species misidentifications and locality errors. Fedotov [1912] reported four species from Novaya Zemlya, which had already been known to occur in that archipelago. Maria Dahl [1928], based on new material collected by the Norwegian expedition to Novaya Zemlya in 1921, published a list of 11 species from numerous localities, including a species she described as new. The latter, however, was synonymized soon after that, but, as shown below, incorrectly. There are no special publications on the spiders of Vaygach, with only a few species recorded from the island by Holm [1973], Eskov [1985, 1988, 1989], Eskov \& Marusik [1994], Tanasevitch \& Nekhaeva [2016] and Tanasevitch [2017].

The objective of the present paper lies in a complete review of the spider faunas of both Novaya Zemlya and Vaygach, based on new material. In addition, a number of old misidentifications, as well as geographical coordinates and locality names are rectified.

\section{Material and methods}

The present paper is largely based on published data, as well as on a fresh material collected in 2013-2016 from Novaya Zemlya and Vaygach by Vitaly M. Spitsyn, Andrei B. Krasheninnikov, Maria V. Gavrilo, Ilia N. Mordvintsev and Nikita G. Platonov. A few specimens kept at the Zoological Museum of the Perm State University (PSU) were kindly sent to me on loan by Sergei L. Esyunin. If not mentioned otherwise, the examined material is deposited in the Zoological Museum of the Moscow State University, Moscow, Russia.

The numbers of the localities (see Map) are given in parentheses.

Abbreviations: a.s.1. — above sea level; A.T. Andrei Tanasevitch; NZ — Novaya Zemlya; S.E Sergei Esyunin.

List of localities in the Novaya Zemlya Archipelago and the Vaygach Island

Note: According to the (sub)zonal regionalization of the Arctic [Matveeva, 1998], localities 19-23 lie within the zone of polar deserts, all the others within the subzone of arctic tundra of the tundra zone.

Novaya Zemlya, Southern Island:

1. Nikitina Bay, $70.562^{\circ} \mathrm{N} 55.225^{\circ} \mathrm{E}$.

2. Cape Kostin Nos, $70.944^{\circ} \mathrm{N} 53.092^{\circ} \mathrm{E}=$ Kostin Skar, Nekhvatova River [Ehlers, 1873a, b; Heuglin, $1874]=$ Kostin Schar (M. Kostin Nos) [Holm, 1973:
72, No. 113].

3. Pomorka (Pomorskaya) Bay, $71.432^{\circ} \mathrm{N}$ $52.852^{\circ} \mathrm{E}=$ Pomorskaja Bucht $=$ Pomork Bucht $[\mathrm{M}$. Dahl, 1928].

4. Cape Yuzhnyi Gusinyi Nos, $71.458^{\circ} \mathrm{N} 51.922^{\circ} \mathrm{E}$ = Södra Gåskap (M. Juz. Gustinyi Nos) [Holm, 1973: 72, No. 109].

5. Cape Severnyi Gusinyi Nos, $72.146^{\circ} \mathrm{N} 51.859^{\circ} \mathrm{E}$ = Norra Gåskap (M. Sev. Gusinyi Nos) [Holm, 1973: 72, No. 36-38].

6. Malye Karmakuly, $72.373^{\circ} \mathrm{N} 52.716^{\circ} \mathrm{E}=$ Möller (Moller) Bay \& Lilla Karmakul Bay [Holm, 1973: 72, No. $43 \& 50]$.

7. Bezymyannaya Bay, $72.895^{\circ} \mathrm{N} 53.197^{\circ} \mathrm{E}=\mathrm{Be}-$ simannaja Bay [Holm, 1973: 72, No. 61, 63, 68].

8. Gribovaya Bay \& Vesyologo Island, $73.015^{\circ} \mathrm{N}$ $53.306^{\circ} \mathrm{E}=$ Gribovii Fjord \& Vesselago Insel [M. Dahl, 1928].

9. Matochkin Shar Strait (western entrance), $73.305^{\circ} \mathrm{N} 54.145^{\circ} \mathrm{E}=$ Chalkonik Tal [M. Dahl, 1928]; Matotschkin Schar [Holm, 1973: 72, No. 71; $73.305^{\circ} \mathrm{N}$ $54.145^{\circ} \mathrm{E}$; No. $75 \& 89$ lie between $73.305^{\circ} \mathrm{N} 54.145^{\circ} \mathrm{E}$ and $\left.73.372^{\circ} \mathrm{N} 54.563^{\circ} \mathrm{E}\right]$.

10. Lazareva Mts, $73.388^{\circ} \mathrm{N} 54.937^{\circ} \mathrm{E}=$ Lasareff Gebirge [M. Dahl, 1928].

Novaya Zemlya, Northern Island:

11. Tyuleniy Bay, $73.339^{\circ} \mathrm{N} 56.015^{\circ} \mathrm{E}=$ Tyouleni Bucht [M. Dahl, 1928].

12. Serebryanka Bay, $73.455^{\circ} \mathrm{N} 54.406^{\circ} \mathrm{E}=$ Serebrjanka Fjord, nördlich vom Lütke Gebirge [M. Dahl, 1928].

13. Cape Sukhoi Nos, $73.784^{\circ} \mathrm{N} 53.720^{\circ} \mathrm{E}=$ Sukhoi Noss [M. Dahl, 1928].

14. Tsyvolki Bay, $74.429^{\circ} \mathrm{N} 58.674^{\circ} \mathrm{E}=$ Zivolka Fjord [M. Dahl, 1928].

15. Mashigina Bay, $74.713^{\circ} \mathrm{N} 56.162^{\circ} \mathrm{E}=$ Maschigin Bucht/Fjord; Sol Bucht \& Dal Bucht [M. Dahl, 1928].

16. Blaafjell Island, $74.656^{\circ} \mathrm{N} 56.738^{\circ} \mathrm{E} \&$ Mt Dietrichson, $74.701^{\circ} \mathrm{N} 56.738^{\circ} \mathrm{E}=$ Blaafjell Insel \& Dietrichson Gebirge [M. Dahl, 1928].

17. Straumsen Bay, $74.754^{\circ} \mathrm{N} 56.451^{\circ} \mathrm{E}=$ Straumsnes (sic!) Bucht [M. Dahl, 1928].

18. Admiralteystva Peninsula, $75.064^{\circ} \mathrm{N} 56.088^{\circ} \mathrm{E}$ = Admiralitätshalb Insel [M. Dahl, 1928].

19. Arkhangel'skaya Bay, $75.855^{\circ} \mathrm{N} 59.095^{\circ} \mathrm{E} \&$ Lichyutina Island, $75.888^{\circ} \mathrm{N} 59.168^{\circ} \mathrm{E}=$ Arkhangel Bucht \& Litchutin Insel [M. Dahl, 1928].

20. Krestovye islands, $76.026^{\circ} \mathrm{N} 59.521^{\circ} \mathrm{E}=$ Krestovii Inseln [M. Dahl, 1928].

21. Pankratieva Peninsula, $76.044^{\circ} \mathrm{N} 60.397^{\circ} \mathrm{E}=$ Pankratjeff Halbinsel [M. Dahl, 1928].

22. Russkaya Gavan' Bay, $76.196^{\circ} \mathrm{N} 62.621^{\circ} \mathrm{E}$.

23. Cape Zhelaniya, $76.950^{\circ} \mathrm{N} 68.545^{\circ} \mathrm{E}$. Island:

Vaygach (= Vaigach, Waigach, Wajgatsch, auct.)

24. Cape Bolvanskiy Nos, $70.454^{\circ} \mathrm{N} 59.058^{\circ} \mathrm{E}$.

25. Yugorskiy Shar $=$ Jugor Schar, northern shore 


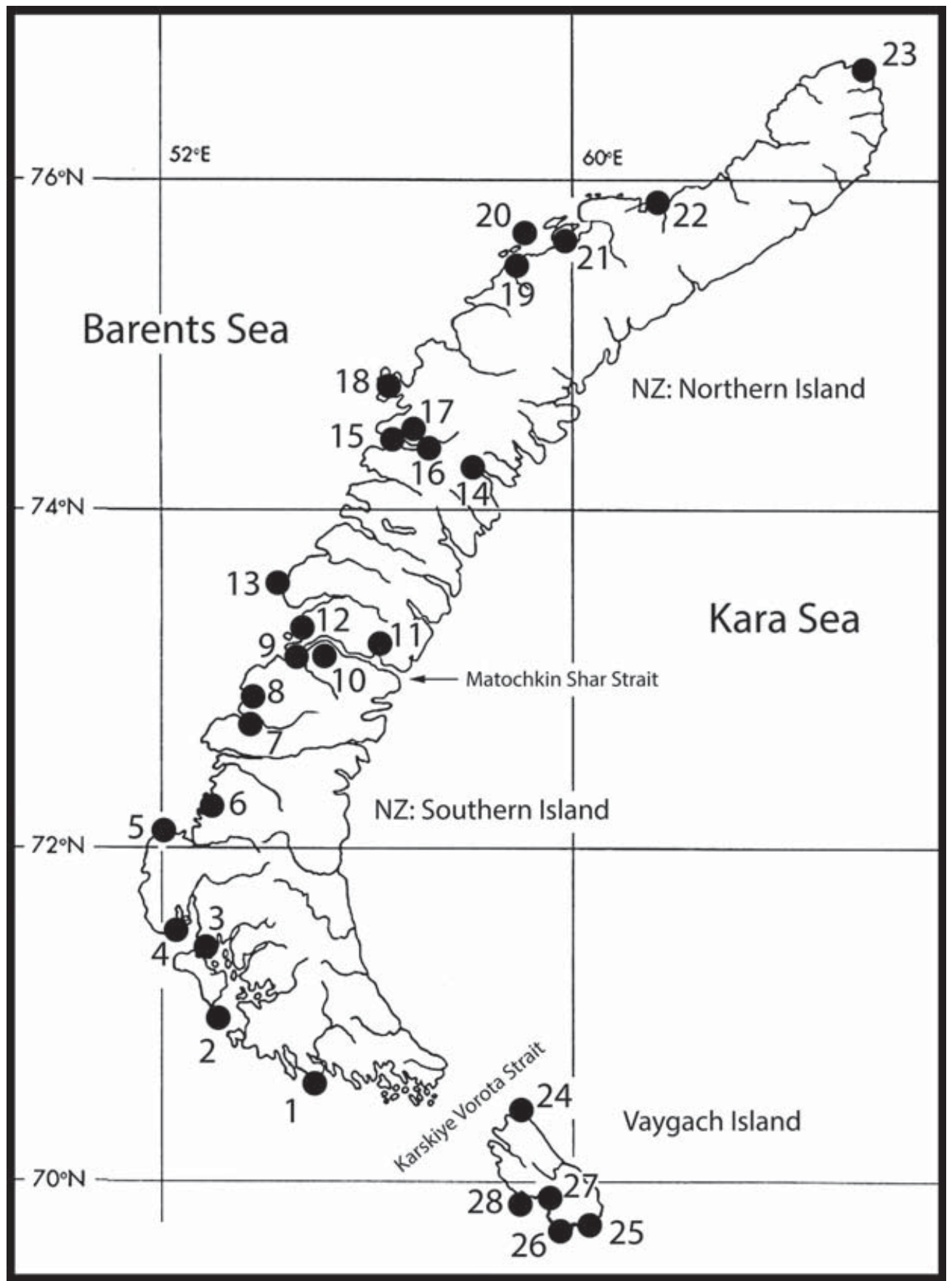

Map. Localities in the Novaya Zemlya Archipelago and the Vaygach Island. Novaya Zemlya (NZ), Southern Island: 1 — Nikitina Bay; 2 - Cape Kostin Nos; 3 - Cape Pomorka (Pomorskaya) Bay; 4 - Yuzhnyi Gusinyi Nos; 5 - Cape Severnyi Gusinyi Nos; 6 - Malye Karmakuly; 7 - Bezymyannaya Bay; 8 - Gribovaya Bay \& Vesyologo Island; 9 - Matochkin Shar Strait; 10 - Lazareva Mts. Novaya Zemlya, Northern Island: 11 - Tyuleniya Bay; 12 - Serebryanka Bay; 13 - Cape Sukhoi Nos; 14 - Tsyvolki Bay; 15 - Mashigina Bay; 16 - Blaafjell Island \& Mt Ditrihson; 17 - Straumsen Bay; 18 - Admiralteystva Peninsula; 19 - Arkhangel'skaya Bay \& Lichyutina Island; 20 - Krestovye islands; 21 - Pankratieva Peninsula; 22 - Russkaya Gavan’ Bay; 23 - Cape Zhelaniya. Vaygach Island: 24 - Cape Bolvanskiy Nos; 25 - Yugorskiy Shar; 26 - Cape Greben' \& Varnek Village; 27 - Okhotnichiya Bay \& Talata River; 28 - Lyamchin Peninsula \& Cape Bol'shoy Lyamchin Nos.

Карта. Точки сборов на архипелаге Новая Земля и о-ве Вайгач. Новая Земля, о-в Южный: 1 — залив Никитина; 2 - пролив Костин Нос; 3 - губа Поморка (Поморская); 4 - мыс Южный Гусиный Нос; 5 - мыс Северный Гусиный Нос; 6 - Малые Кармакулы; 7 - губа Безымянная; 8 - губа Грибовая и о-в Весёлого; 9 - пролив Маточкин Шар; 10 - горы Лазарева. Новая Земля, о-в Северный: 11 - Тюлений залив; 12 - губа Серебрянка; 13 - мыс Сухой Нос; 14 - залив Цивольки; 15 - губа Машигина; 16 - о-в Блафьеля и гора Дитрихсона; 17 - бухта Штраумсена; 18 - п-ов Адмиралтейства; 19 - губа Архангельская и о-в Личутина; 20 - о-ва Крестовые; 21 - п-ов Панкратьева; 22 - залив Русская Гавань; 23 - мыс Желания. Остров Вайгач: 24 - мыс Болванский Нос; 25 - Югорский Шар; 26 - мыс Гребень и пос. Варнек; 27 - губа Охотничья и р. Талата; 28 - п-ов Лямчин и мыс Большой Лямчин Нос. 
[Holm, 1973: 72, No. 137; 69.683 $\mathrm{N} 60.286^{\circ} \mathrm{E} \&$ No. $\left.142 ; 69.699^{\circ} \mathrm{N} 60.384^{\circ} \mathrm{E}\right]$.

26. Cape Greben', $69.663^{\circ} \mathrm{N} 59.985^{\circ} \mathrm{E} \&$ Varnek, $69.710^{\circ} \mathrm{N} 60.064^{\circ} \mathrm{E}=$ Cape Grebeni [Holm, 1973: 72, No. 129].

27. Okhotnichya Bay, $69.8842^{\circ} \mathrm{N} 59.357^{\circ} \mathrm{E} \&$ Talata River, $69.824^{\circ} \mathrm{N} 59.654^{\circ} \mathrm{E}$.

28. Lyamchin Peninsula \& Cape Bol'shoy Lyamchin Nos, $69.856^{\circ} \mathrm{N} 59.168^{\circ} \mathrm{E}$.

\section{List of the Novaya Zemlya spiders}

Fam. Linyphiidae

Agyneta nigripes (Simon, 1884) (?)

1879 Erigone rurestris non C.L. Koch. - L. Koch: 40, in part sensu Holm [1973].

1973 Meioneta nigripes. - Holm: 97.

REMARKS. IN NZ, the species has been recorded from the Bezymyannaya Bay (No. 7) and Matochkin Shar (No. 9) [L. Koch, 1879; Holm, 1973]. In both habitats, the species has been identified based on females only, but in the absence of males, the determination cannot be considered as reliable.

Agyneta similis (Kulczyński, 1926)

1928 Microneta rurestris. - M. Dahl: 23, figs 36-41, o' \& + , misidentification.

1994 Agyneta nigripes. — Eskov, Marusik: 68, misidentification.

REMARKS. In NZ, the species has been referred to as Microneta rurestris from the Pomorskaya Bay (No. 3) and the Blaafjell Island (No. 16) by M. Dahl [1928]. Later, Eskov \& Marusik [1994: 68] considered that record as a mistake, and attributed it to $A$. nigripes. However, the record of Microneta rurestris was accompanied by a drawing of its palp [M. Dahl, 1928, fig. 37]. The drawing, namely the shape of the lamella characteristica (in dorsal view) undoubtedly shows $A$. similis, a vicariant species of $A$. rurestris in the northern and eastern regions of the Palaearctic.

Agyneta similis is new to the fauna of NZ.

\section{Arcterigone pilifrons (L. Koch, 1879)}

NEW MATERIAL EXAMINED. $1 \sigma^{7}, 1$, NZ, Malye Karmakuly (No. 6), environs of Polar Station, $72.382200^{\circ} \mathrm{N}$ $52.750550^{\circ} \mathrm{E}$, wet Saxifraga-Rhodiola rosea communities, with Sedum roseum, Saxifraga hirculus, S. cespitosa, S. cernua, Myosotis asiatica, mosses, pitfall traps, 21.VII.-1.VIII. 2015, leg. V. Spitsyn, det. A.T.; 1 ' , same locality, environs of Polar Station, $72.375413^{\circ} \mathrm{N} 52.724130^{\circ} \mathrm{E}$, sedge-Sphagnum tundra with Polemonium \& Artemisia tilesii and dwarf willow-moss communities on microelevations, pitfall traps, 21.VII.-1.VIII.2015, leg. V. Spitsyn, det. A.T.; 2 우, same locality, environs of Polar Station, $72.379683^{\circ} \mathrm{N} 52.752529^{\circ} \mathrm{E}$, small-hummocky tundra, with dwarf willow-moss-sedge tundra, pitfall traps, 22.VII.-1.VIII.2015, leg. V. Spitsyn, det. A.T.; $5 \sigma^{7} \sigma^{7}, 8$ 우, same locality, environs of Polar Station, $72.347051^{\circ} \mathrm{N} 52.806266^{\circ} \mathrm{E}$, wet small-hummocky dwarf willow-moss-sedge tundra, with Eriophorum scheuchzeri, Carex sp., Salix arctica, S. polaris, mosses, pitfall traps, 18-31.VII.2015, leg. V. Spitsyn, det. A.T.
REMARKS. The species is new to the fauna of NZ.

Diplocephalus barbiger (Roewer, 1955)

1879 Erigone incerta. - L. Koch: 52, T. 2, f. 6, 6a, ㅇ. 1879 E. barbata. - L. Koch: 60, T. 2, f. 13, 13a, o’ \&.+ 1928 Savignia barbata. - M. Dahl: 18, figs 27-28, ㅇ. 1973 Diplocephalus barbatus. - Holm: 81.

NEW MATERIAL EXAMINED. $1 \sigma^{7}, 2$ 우, NZ, Malye Karmakuly (No. 6), environs of Polar Station, $72.382200^{\circ} \mathrm{N}$ $52.750550^{\circ} \mathrm{E}$, wet Saxifraga-Rhodiola rosea communities, with Sedum roseum, Saxifraga hirculus, S. cespitosa, S. cernua, Myosotis asiatica, mosses, pitfall traps, 21.VII.-1.VIII. 2015, leg. V. Spitsyn, det. A.T.; 2 ㅇ, same locality, environs of Polar Station, $72.379683^{\circ} \mathrm{N} 52.752529^{\circ} \mathrm{E}$, small-hummocky tundra, with dwarf willow-moss-sedge tundra, pitfall traps, 22.VII.-1.VIII.2015, leg. V. Spitsyn, det. A.T.

REMARKS. In NZ, this species has been recorded from the Blaafjell Island (No. 16), the Mashigina Bay (No. 15) [M. Dahl, 1928], Malye Karmakuly (No. 6), the Bezymyannaya Bay (No. 7), and the Cape Yuzhnyi Gusinyi Nos (No. 4) [L. Koch, 1879; Holm, 1973].

Erigone arctica palaearctica Braendegaard, 1934

1873a Erigone longipalpis. — Ehlers: 7, misidentification or unreliable record sensu Holm [1973].

1873 b E. longipalpis. - Ehlers: 465, misidentification or unreliable record sensu Holm [1973].

1874 E. longipalpis. - Heuglin: 236, misidentification or unreliable record sensu Holm [1973].

1879 E. arctica. — L. Koch: 39, in part sensu Holm [1973].

1912 E. arctica. - Fedotov: 457, fig. 5,, .

1928 E. longipalpis. - M. Dahl: 11, figs 16, 18, , misidentification.

1973 E. arctica palaearctica. - Holm: 82

NEW MATERIAL EXAMINED. $2 \sigma^{\top} \sigma^{\top}, 1$ ㅇ, NZ, Nikitina Bay (No. 1), swampy bank of brook, $70.581509^{\circ} \mathrm{N} 55.060510^{\circ} \mathrm{E}, 13 \mathrm{~m}$ a.s.1., 27.VII.2015, leg. A. Krasheninnikov, det. S.E. (as E. a. ?sibirica); 1 을 same locality, brook in tundra, $70.551933^{\circ} \mathrm{N} 55.206440^{\circ} \mathrm{E}$, $12 \mathrm{~m}$ a.s.1., 28.VII.2015, leg. A. Krasheninnikov, det. S.E. (as E. $a$. ?sibirica); 19 , same locality, brook in tundra, $70.578701^{\circ} \mathrm{N}$ $55.212207^{\circ} \mathrm{E}, 56 \mathrm{~m}$ a.s.1., 2.VIII.2015, leg. A. Krasheninnikov, det. S.E.; 3 우, same locality, bank of lake, $70.588492^{\circ} \mathrm{N} 55.044716^{\circ} \mathrm{E}$, $35 \mathrm{~m}$ a.s.1., 5.VIII.2015, leg. A. Krasheninnikov, det. S.E. (as E. a.

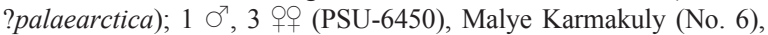
26.VIII.2014, leg. M. Gavrilo, det. S.E.; $7 \sigma^{7} \sigma^{7}, 15$ ㅇ, same locality, environs of Polar Station, $72.382200^{\circ} \mathrm{N} 52.750550^{\circ} \mathrm{E}$, wet Saxifraga-Rhodiola rosea communities, with Sedum roseum, Saxifraga hirculus, S. cespitosa, S. cernua, Myosotis asiatica, mosses, pitfall traps, 21.VII.-1.VIII. 2015, leg. V. Spitsyn, det. A.T.; $4 \bigcirc^{7} \sigma^{7}, 4$ 우, same locality, environs of Polar Station, $72.361884^{\circ} \mathrm{N} 52.772449^{\circ} \mathrm{E}$, dry Dryas-dwarf willow rocky tundra, pitfall traps, 17-31.VII.2015, leg. V. Spitsyn, det. A.T.; 1 O$^{7}, 2$ 우, Polar Station, $72.373867^{\circ} \mathrm{N}$ $52.716733^{\circ} \mathrm{E}$, gravelly plots with Artemisia borealis, among stones, 9.VIII.2015, leg. V. Spitsyn, det. A.T.; $3 \sigma^{7} \sigma^{7}$, same locality, environs of Polar Station, $72.375413^{\circ} \mathrm{N} 52.724130^{\circ} \mathrm{E}$, sedge-Sphagnum tundra with Polemonium \& Artemisia tilesii and dwarf willow-moss communities on microelevations, pitfall traps, 21.VII.-1.VIII.2015, leg. V. Spitsyn, det. A.T.

REMARKS. In NZ, the species has been recorded from the Cape Kostin Nos, Nekhvatova River (No. 2) [Ehlers, 1873a, b; Heuglin, 1874], Malye Karmakuly (No. 6) [L. Koch, 1879; Holm, 1973]; from NZ, without precise locality [Fedotov, 1912], as well as the Mashigina Bay (No. 15) [M. Dahl, 1928] (see below under E. remota). 
Erigone arcticola Chamberlin et Ivie, 1947

1879 Erigone arctica non White. — L. Koch: 39, misidentification, in part sensu Holm [1973].

1879 E. atra non Blackwall. - L. Koch: 40, misidentification, in part sensu Holm [1973].

1879 E. remota non L. Koch. — L. Koch: 40, misidentification, in part sensu Holm [1973].

1973 E. arctophylacis. - Holm: 82, fig. 35, , misidentification sensu Crawford [1988].

REMARKS. I fully agree with Crawford [1988] that the vulva drawn by Holm [1973] belongs to $E$. arcticola, not to E. arctophylacis Crosby et Bishop, 1928. So the latter species is to be ejected from the Russian list, as it occurs in fact only in the Western Nearctic [Paquin et al., 2010].

Erigone arcticola is new to the fauna of NZ.

\section{Erigone psychrophila Thorell, 1872}

1879 Erigone psychrophila. - L. Koch: 47, T. 2, f. 3, 3a-d, $\sigma^{7} \&+$.

1912 E. psychrophila. - Fedotov: 456, fig. 4, + .

1928 E. psychrophila. - M. Dahl: 9, figs 11-14, ơ \&

1928 Macrargus solitarius. - M. Dahl: 26, figs 42-44, 우, syn.n.

1973 E. psychrophila. — Holm: 84.

NEW MATERIAL EXAMINED. $7 \sigma^{7} \sigma^{7}, 6$ 90 , NZ, Malye Karmakuly (No. 6), environs of Polar Station, $72.375413^{\circ} \mathrm{N} 52.724130^{\circ} \mathrm{E}$ sedge-Sphagnum tundra with Polemonium \& Artemisia tilesii and dwarf willow-moss communities on microelevations, pitfall traps, 21.VII.-1.VIII.2015, leg. V. Spitsyn, det. A.T.; 3 O$^{7} \sigma^{7}, 5$ 50 , 3 juv., same locality, environs of Polar Station, $72.379683^{\circ} \mathrm{N} 52.752529^{\circ} \mathrm{E}$, small-hummocky tundra, with dwarf willow-moss-sedge tundra, pitfall traps, 22.VII.-1.VIII.2015, leg. V. Spitsyn, det. A.T.; $1 O^{7}, 1$, same locality, environs of Polar Station, $72.347051^{\circ} \mathrm{N} 52.806266^{\circ} \mathrm{E}$, wet small-hummocky dwarf willow-moss-sedge tundra, with Eriophorum scheuchzeri, Carex sp., Salix arctica, S. polaris, mosses, pitfall traps, 18-31.VII.2015, leg. V. Spitsyn, det. A.T.; 2 o Russkaya Gavan' Bay (No. 22), bank of brook, $76.19041^{\circ} \mathrm{N}$ $62.64605^{\circ} \mathrm{E}, 4 \mathrm{~m}$ a.s.1., under stones, 5.VIII.2016, leg. A. Krasheninnikov, det. S.E.; $1 \Im^{7}$, same locality, middle flow of brook, $76.18721^{\circ} \mathrm{N}$ $62.66831^{\circ} \mathrm{E}, 10 \mathrm{~m}$ a.s.1., under stones on bank, 5.VIII.2016, leg. A. Krasheninnikov, det. S.E.; 2 9 , same locality, Melkoye Lake, $76.18435^{\circ} \mathrm{N} 62.69544^{\circ} \mathrm{E}$, under stones, 5.VIII.2016, leg. A. Krasheninnikov, det. S.E.; 1 (PSU-6444), Cape Zhelaniya (No. 23), Snezhnaya River flow, 2013, leg. M. Gavrilo, det. S.E.

REMARKS. In NZ, the species has been recorded from numerous localities: No. 2-5, 7, 9, 11, 13-16, 19-21 [L. Koch, 1879; Fedotov, 1912; M. Dahl, 1928; Holm, 1973].

Maria Dahl [1928] described Macrargus solitarius M. Dahl, 1928, based on a single female from the Pankratieva Peninsula (No. 21). The description was supplied with a drawing of the epigyne, which in fact belongs to Erigone psychrophila, not to Masikia indistincta (Kulczyński, 1908), as mentioned by Eskov \& Marusik [1994: 47]. Thus, Macrargus solitarius is to be considered as a junior synonym of E. psychrophila, not of $M$. indistincta (syn.n.).

\section{Erigone remota L. Koch, 1869} 1973]

1879 Erigone remota. - L. Koch: 40, in part sensu Holm 1928 E. longipalpis. - M. Dahl: 11, figs 15, 17, ○’, misidentification.

1973 E. remota. - Holm: 84.
NEW MATERIAL EXAMINED. 2 90 (PSU-6451), NZ, Malye Karmakuly (No. 6), 26.VIII.2014, leg. M. Gavrilo, det. S.E.

REMARKS. In NZ, the species has been recorded from the Cape Yuzhnyi Gusinyi Nos (No. 4) and the Bezymyannaya Bay (No. 7) [L. Koch, 1879; Holm, 1973]. The record of Erigone longipalpis (Sundevall, 1830) from the Mashigina Bay (No. 15) [M. Dahl, 1928] is based on a misidentification: according to her drawings, the depicted palp actually represents $E$. re$m o t a$, but the cleared epigyne undoubtedly belongs to E. arctica palaearctica (see above).

Erigone tirolensis L. Koch, 1872 [1973]

1879 Erigone tirolensis. - L. Koch: 39, in part sensu Holm

1879 E. arctica (non White). - L. Koch: 39, misidentification, in part sensu Holm [1973].

1973 E. tirolensis. — Holm: 84.

NEW MATERIAL EXAMINED. $1 \sigma^{7}$, NZ, Malye Karmakuly (No. 6), Polar Station, $72.373867^{\circ} \mathrm{N} 52.716733^{\circ} \mathrm{E}$, gravelly plots with Artemisia borealis, among stones, 9.VIII.2015, leg. V. Spitsyn, det. A.T.

REMARKS. In NZ, the species has been recorded from the Bezymyannaya Bay (No. 7) and the Matochkin Shar Strait (No. 9) [L. Koch, 1879; Holm, 1973].

\section{Gibothorax tchernovi Eskov, 1989}

NEW MATERIAL EXAMINED. $3 \sigma^{\top} \sigma^{\top}, 2$ o, , NZ, Malye Karmakuly (No. 6), environs of Polar Station, $72.382200^{\circ} \mathrm{N}$ $52.750550^{\circ} \mathrm{E}$, wet Saxifraga-Rhodiola rosea communities, with Sedum roseum, Saxifraga hirculus, $S$. cespitosa, S. cernua, Myosotis asiatica, mosses, pitfall traps, 21.VII.-1.VIII. 2015, leg. V. Spitsyn, det. A.T.; $23 \bigcirc^{7} \sigma^{7}, 8$ 옹, same locality, environs of Polar Station, $72.375413^{\circ} \mathrm{N} 52.724130^{\circ} \mathrm{E}$, sedge-Sphagnum tundra with Polemonium \& Artemisia tilesii and dwarf willow-moss communities on microelevations, pitfall traps, 21.VII.-1.VIII.2015, leg. V. Spitsyn, det. A.T

REMARKS. The species is new to the fauna of NZ.

Halorates boreus (L. Koch, 1879)

1879 Erigone borea. - L. Koch: 40, T. 1, f. 28, 28a-d, ơ \&

1973 Collinsia borea. - Holm, 1973: 79, figs 11-18, or \& ㅇ.

REMARKS. In NZ, the species has been recorded from the Bezymyannaya Bay (No. 7) [L. Koch, 1879; Holm, 1973].

\section{Halorates holmgreni (Thorell, 1871)}

1879 Erigone mendica. - L. Koch: 52, T. 2, f. 7, 7a-c, ○ \& 우. $26 \mathrm{a}-\mathrm{c}$,,

Microerigone mendica. - M. Dahl: 17, figs 23-25,

1973 Collinsia holmgreni. - Holm: 79.

NEW MATERIAL EXAMINED. $3 \sigma^{\top} \sigma^{7}, 8$ 우, NZ, Malye Karmakuly (No. 6), Polar Station, $72.373867^{\circ} \mathrm{N} 52.716733^{\circ} \mathrm{E}$, gravelly plots with Artemisia borealis, among stones, 9.VIII.2015, leg. V. Spitsyn, det. A.T.; $2 \bigcirc^{7} \sigma^{7}, 1$, same locality, environs of Polar Station, $72.382200^{\circ} \mathrm{N} 52.750550^{\circ} \mathrm{E}$, wet Saxifraga-Rhodiola rosea communities, with Sedum roseum, Saxifraga hirculus, S. cespitosa, S. cernua, Myosotis asiatica, mosses, pitfall traps, 21.VII.-1.VIII. 2015, leg. V. Spitsyn, det. A.T.; 2 O $^{7} \sigma^{7}, 4$ 우 same locality, environs of Polar Station, $72.375413^{\circ} \mathrm{N} 52.724130^{\circ} \mathrm{E}$, sedge-Sphagnum tundra with Polemonium \& Artemisia tilesii and dwarf willow-moss communities on microelevations, pitfall traps, 
21.VII.-1.VIII.2015, leg. V. Spitsyn, det. A.T.; 1 , same locality, environs of Polar Station, $72.379683^{\circ} \mathrm{N} 52.752529^{\circ} \mathrm{E}$, small-hummocky tundra, with dwarf willow-moss-sedge tundra, pitfall traps, 22.VII.-1.VIII.2015, leg. V. Spitsyn, det. A.T.; 1 O’, 3 +P (PSU6450), same locality, 26.VIII.2014, leg. M. Gavrilo, det. S.E.

REMARKS. In NZ, the species has been recorded from the Pomorka Bay (No. 3), the Lichyutina Island (No. 19) [M. Dahl, 1928], the Cape Kostin Nos (No. 2), Malye Karmakuly (No. 6), the Bezymyannaya Bay (No. 7) and the Matochkin Shar Strait (No. 9) [L. Koch, 1879; Holm, 1973].

\section{Halorates spetsbergensis (Thorell, 1872)} $\&$ ㅇ.

1879 Erigone oxycephala. - L. Koch: 54, T. 2, f. 8, 8a-d, o7

1912 Typhochraestus [sic!] spetsbergensis. - Fedotov: 458. $\sigma^{7} \&$.

1928 Microerigone spetsbergensis. - Dahl: 14, figs 19-22,

1973 Collinsia spetsbergensis. - Holm: 81.

NEW MATERIAL EXAMINED. 1 9 , NZ, Russkaya Gavan' Bay (No. 22), in moss, 8.VII.2014, leg. M. Gavrilo, det. S.E.; 2 우, 1 o subad., same locality, Melkoye Lake, $76.18435^{\circ} \mathrm{N} 62.69544^{\circ} \mathrm{E}$ under stones, 5.VIII.2016, leg. A. Krasheninnikov, det. S.E.; 1 ㅇ, Cape Zhelaniya (No. 23), under stones, 24.VII.2014, leg. I. Mordvintsev \& N. Platonov, det. A.T.

REMARKS. In NZ, the species has been recorded from numerous localities: No. 3, 6-9, 11, 13-16, 1821 [L. Koch, 1879; Holm, 1973; Fedotov, 1912; M. Dahl, 1928].

\section{Hilaira glacialis (Thorell, 1871)}

1879 Erigone vexatrix (non O. Pickard-Cambridge). - L. Koch: 45, T.2, f. 2, 2a-e, $\sigma^{7} \&$ 9 , misidentification, in part sensu Holm [1973].

1973 Hilaira glacialis. - Holm: 84.

NEW MATERIAL EXAMINED. $4 \sigma^{7} \sigma^{7}, 13$ ㅇ․, NZ, Malye Karmakuly (No. 6), environs of Polar Station, $72.347051^{\circ} \mathrm{N}$ $52.806266^{\circ} \mathrm{E}$, wet small-hummocky dwarf willow-moss-sedge tundra, with Eriophorum scheuchzeri, Carex sp., Salix arctica, S. polaris, mosses, pitfall traps, 18-31.VII.2015, leg. V. Spitsyn, det. A.T.

REMARKS. In NZ, the species has been recorded from the Cape Kostin Nos (No. 2) and Malye Karmakuly (No. 6) [L. Koch, 1879; Holm, 1973].

\section{Hilaira nivalis Holm, 1937}

NEW MATERIAL EXAMINED. $7 \sigma^{7} \sigma^{7}, 15$ Oᄋ, NZ, Malye Karmakuly (No. 6), environs of Polar Station, $72.382200^{\circ} \mathrm{N}$ $52.750550^{\circ} \mathrm{E}$, wet Saxifraga-Rhodiola rosea communities, with Sedum roseum, Saxifraga hirculus, S. cespitosa, S. cernua, Myosotis asiatica, mosses, pitfall traps, 21.VII.-1.VIII. 2015, leg. V. Spitsyn, det. A.T.; $5 \bigcirc^{\top} \sigma^{7}, 19$ q0, same locality, environs of Polar Station, $72.375413^{\circ} \mathrm{N} 52.724130^{\circ} \mathrm{E}$, sedge-Sphagnum tundra with Polemonium \& Artemisia tilesii and dwarf willow-moss communities on microelevations, pitfall traps, 21.VII.-1.VIII.2015, leg. V. Spitsyn, det. A.T.; $17 \bigcirc^{\top} \sigma^{7}, 53$ 90 , same locality, environs of Polar Station, $72.379683^{\circ} \mathrm{N} 52.752529^{\circ} \mathrm{E}$, small-hummocky tundra, with dwarf willow-moss-sedge tundra, pitfall traps, 22.VII.-1.VIII.2015, leg. V. Spitsyn, det. A.T.; $14 \sigma^{\top} \sigma^{7}, 49$ क+ , same locality, environs of Polar Station, $72.347051^{\circ} \mathrm{N} 52.806266^{\circ} \mathrm{E}$, wet small-hummocky dwarf willow-moss-sedge tundra, with Eriophorum scheuchzeri, Carex sp., Salix arctica, S. polaris, mosses, pitfall traps, 1831.VII.2015, leg. V. Spitsyn, det. A.T.

REMARKS. The species is new to the fauna of NZ.

\section{Hilaira proletaria (L. Koch, 1879)}

NEW MATERIAL EXAMINED. $2 \sigma^{7} \sigma^{7}, 8$ 우, NZ, Malye Karmakuly (No. 6), environs of Polar Station, $72.347051^{\circ} \mathrm{N}$ $52.806266^{\circ} \mathrm{E}$, wet small-hummocky dwarf willow-moss-sedge tundra, with Eriophorum scheuchzeri, Carex sp., Salix arctica, S. polaris, mosses, pitfall traps, 18-31.VII.2015, leg. V. Spitsyn, det. A.T.

REMARKS. The species is new to the fauna of NZ.

\section{Hybauchenidium aquilonare (L. Koch, 1879)}

1879 Erigone aquilonaris. - L. Koch: 42, T. 1, f. 29a-g, O \&

1928 Oedothorax aquilonaris. - M. Dahl: 22, figs 33-35, ơ $\&+$ $\sigma^{\top} \&$.

1973 Hybauchenidium aquilonare. - Holm: 86, figs 38-45,

REMARKS. In NZ, the species has been recorded from the Cape Kostin Nos (No. 2), the Cape Yuzhnyi Gusinyi Nos (No. 4), Malye Karmakuly (No. 6) [L. Koch, 1879; Holm, 1973], the Mashigina Bay (No. 15) and the Blaafjell Island (No. 16) [M. Dahl, 1928].

\section{Masikia indistincta (Kulczyński, 1908)}

NEW MATERIAL EXAMINED. $1 \sigma^{7}, 19$, NZ, Malye Karmakuly (No. 6), environs of Polar Station, $72.382200^{\circ} \mathrm{N}$ $52.750550^{\circ} \mathrm{E}$, wet Saxifraga-Rhodiola rosea communities, with Sedum roseum, Saxifraga hirculus, S. cespitosa, S. cernua, Myosotis asiatica, mosses, pitfall traps, 21.VII.-1.VIII. 2015, leg. V. Spitsyn, det. A.T.; 1 o, same locality, environs of Polar Station, $72.375413^{\circ} \mathrm{N} 52.724130^{\circ} \mathrm{E}$, sedge-Sphagnum tundra with Polemonium \& Artemisia tilesii and dwarf willow-moss communities on microelevations, pitfall traps, 21.VII.-1.VIII.2015, leg. V. Spitsyn, det. A.T.

REMARKS. The species is new to the fauna of NZ.

Mughiphantes sobrius (Thorell, 1871) $\&$ \&.

1879 Linyphia latebricola. - L. Koch: 19, T. 1, f. 7, 7a-e, o7

1912 Lephthyphantes [sic!] latebricola. - Fedotov: 459

1928 Lephthyphantes latebricolus [sic!]. - M. Dahl: 6, figs $5-10,0^{7} \&$ ㅇ․

1973 Lepthyphantes sobrius. - Holm: 95.

REMARKS. In NZ, the species has been recorded from numerous localities: No. 3, 5, 7, 9, 10, 12, 17, 19 [L. Koch, 1879; Fedotov, 1912; M. Dahl, 1928; Holm, 1973].

\section{Oreoneta leviceps (L. Koch, 1879)}

1879 Erigone leviceps. — L. Koch: 63, T. 2, f. 15, 15a-c, O’ \& ㅇ․

1928 Hilaira leviceps. - M. Dahl: 21, figs 29-32, $0^{7} \&$ 우

1973 H. leviceps. - Holm: 85.

NEW MATERIAL EXAMINED. $1{ }^{\top}, 1$, , NZ, Malye Karmakuly (No. 6), environs of Polar Station, $72.382200^{\circ} \mathrm{N}$ $52.750550^{\circ} \mathrm{E}$, wet Saxifraga-Rhodiola rosea communities, with Sedum roseum, Saxifraga hirculus, S. cespitosa, S. cernua, Myosotis asiatica, mosses, pitfall traps, 21.VII.-1.VIII. 2015, leg. V. Spitsyn, det. A.T.; 1 , environs of Polar Station, $72.361884^{\circ} \mathrm{N}$ $52.772449^{\circ} \mathrm{E}$, dry Dryas-dwarf willow rocky tundra, pitfall traps, 17-31.VII.2015, leg. V. Spitsyn, det. A.T.; $10^{\top}, 2$ ㅇ, same locality, environs of Polar Station, $72.347051^{\circ} \mathrm{N} 52.806266^{\circ} \mathrm{E}$, wet smallhummocky dwarf willow-moss-sedge tundra, with Eriophorum 
scheuchzeri, Carex sp., Salix arctica, S. polaris, mosses, pitfall traps, 18-31.VII.2015, leg. V. Spitsyn, det. A.T.

REMARKS. In NZ, the species has been recorded from the Pomorka Bay (No. 3), the Matochkin Shar Strait (No. 9) [L. Koch, 1879; M. Dahl, 1928; Holm, 1973], the Cape Kostin Nos (No. 2), Malye Karmakuly (No. 6) and the Bezymyannaya Bay (No. 7) [L. Koch, 1879; Holm, 1973].

Fam. Theridiidae

Thymoites oleatus (L. Koch, 1879)

1879 Theridium oleatum. — L. Koch: 81, T. 3, f. 2, 2a, $\sigma^{7}$ \& 9 1928 T. oleatum. - M. Dahl: 4, figs 1-4, $0^{\top} \&$ 9 .

1973 Thymoites oleatus. - Holm: 78.

REMARKS. In NZ, the species has been recorded from the Pomorka Bay (No. 3), the Straumsen Bay (No. 17) [M. Dahl, 1928] and Malye Karmakuly (No. 6) [L. Koch, 1879; Holm, 1973].

\section{Misplaced species} lya:

Species erroneously recorded from Novaya Zem-

1. Holm [1973: 103] considered the female-based record of Haplodrassus cognatus (Westring, 1861) by L. Koch [1879: 87, as Drassus c.] from the "N. Gåskap" as a mislabeled specimen.

2. Concerning Pirata piraticus (Clerck, 1758), Braendegaard [1958] wrote: "but while L. Koch states that it was taken at "Gåskap" on Novaya Zemlya, the label in the tube is marked: "Vorogowo Selo", lat. $60^{\circ}$ $50^{\prime}$. The village of Vorogovo is situated in Middle Siberia $\left(61.025488^{\circ} \mathrm{N} 89.616537^{\circ} \mathrm{E}\right)$.

3. L. Koch [1879] gave records of three species, viz. Robertus lividus (Blackwall, 1836), as Erigone; Pardosa palustris (Linnaeus, 1758) and Zelotes subterraneus (C.L. Koch, 1833), as Prosthesima petiveri (Scopoli, 1763) from "Fumkn Remon" or "Funken Renon". Subsequent authors have conceived these as locality names from West Siberia or Novaya Zemlya. They are, however, misinterpretations of "Finmkn, Renön" on the labels which means the island Reinöy in the Norwegian Finnmark [cit. by Holm, 1973: 76].

\section{List of the Vaygach Island spiders}

Agyneta bulavintsevi Tanasevitch, 2016

2009 Agyneta sp. 1. - Marusik, Eskov: 138. (?).

2016 A. bulavintsevi. — Tanasevitch, Nekhaeva: 314

REMARKS. On the Vaygach Island, the species has been recorded from the Cape Bolvanskiy Nos (No. 24) [Tanasevitch, Nekhaeva, 2016]. Agyneta bulavintsevi seems to have been reported from the island as Agyneta sp. 1. [Marusik, Eskov, 2009: 138]; these authors also noted it as "a species with an unclear taxonomic status".
Agyneta nigripes (Simon, 1884)

1879 Erigone rurestris non C.L. Koch. - L. Koch: 40, misidentification, in part sensu Holm [1973].

1973 Meioneta nigripes. — Holm: 97.

1994 Agyneta nigripes. - Eskov, Marusik: 69

REMARKS. On the Vaygach Island, the species has been recorded from the Cape Greben' (No. 26) [L. Koch, 1879; Holm, 1973] and Talata River (No. 27) [Eskov, Marusik, 1994]. The species identification is based on males and can be considered as reliable.

\section{Bathyphantes humilis (L. Koch, 1879)}

2017 Bathyphantes humilis. — Tanasevitch: 78.

REMARKS. On the Vaygach Island, the species has been recorded from Varnek (No. 26) [Tanasevitch, 2017].

\section{Bathyphantes gracilis (Blackwall, 1841)}

NEW MATERIAL EXAMINED. 2 우, Vaygach Island, environs of Okhotnichiya Bay (No. 27), swampy bank, $69.884208^{\circ} \mathrm{N}$ $59.357198^{\circ} \mathrm{E}, 1 \mathrm{~m}$ a.s.1., 7.VIII.2015, leg. A. Krasheninnikov, det. S.E.; $1 \bigcirc^{\top}, 2$ 우, Lyamchin Peninsula, environs of Cape Bol'shoy Lyamchin Nos (No. 28), middle flow of brook, $69.860027^{\circ} \mathrm{N}$ 59. $133420^{\circ}$ E, $10 \mathrm{~m}$ a.s.1., 7.VIII.2015, leg. A. Krasheninnikov, det. S.E.

REMARKS. The species is new to the fauna of the Vaygach Island.

\section{Diplocephalus barbiger (Roewer, 1955)}

1879 Erigone incerta. - L. Koch: 52, T. 2, f. 6, 6a, ․

1879 E. barbata. - L. Koch: 60, T. 2, f. 13, 13a, o' \& ㅇ․

1973 Diplocephalus barbatus. - Holm: 81.

1988 D. barbatus. - Eskov: 16.

REMARKS. On the Vaygach Island, the species has been recorded from Yugorsky Shar (No. 25), the Cape Greben' (No. 26) [L. Koch, 1879; Holm, 1973], the Cape Bolvanskiy Nos (No. 24) and Talata River (No. 27) [Eskov, 1988].

Erigone arctica palaearctica Braendegaard, 1934 [1973]

1879 Erigone arctica. - L. Koch: 39, in part sensu Holm

1973 E. arctica palaearctica. - Holm: 82.

REMARKS. On the Vaygach Island, the species has been recorded from Yugorsky Shar (No. 25) and the Cape Greben' (No. 26) [L. Koch, 1879; Holm, 1973].

Erigone psychrophila Thorell, 1872 $\sigma^{7} \& 9$

1879 Erigone psychrophila. - L. Koch: 47, T. 2, f. 3, 3a-d,

1973 E. psychrophila. — Holm: 84.

1985 E. psychrophila. - Eskov: 124.

REMARKS. On the Vaygach Island, the species has been recorded from Yugorsky Shar (No. 25) [L. Koch, 1879; Holm, 1973] and the Cape Bolvanskiy Nos (No. 24) [Eskov, 1985]. 
Erigone tirolensis L. Koch, 1872

1994 Erigone tirolensis. - Eskov, Marusik: 71.

REMARKS. On the Vaygach Island, the species has been recorded from Talata River (No. 27) [Eskov, Marusik, 1994].

\section{Halorates holmgreni (Thorell, 1871)}

1879 Erigone mendica. — L. Koch: 52, T. 2, f. 7, 7a-c, $0^{7} \&$ ㅇ. 1973 Collinsia holmgreni. - Holm: 79.

1985 C. holmgreni. - Eskov: 123.

REMARKS. On the Vaygach Island, the species has been recorded from Yugorsky Shar (No. 25) and the Cape Bolvanskiy Nos (No. 24) [Eskov, 1985].

Halorates spetsbergensis (Thorell, 1872) $\&$ 우

1879 Erigone oxycephala. - L. Koch: 54, T. 2, f. 8, 8a-d, o7

1973 Collinsia spetsbergensis. - Holm: 81.

REMARKS. On the Vaygach Island, the species has been recorded from Yugorsky Shar (No. 25) and the Cape Greben' (No. 26) [L. Koch, 1879; Holm, 1973].

\section{Hilaira glacialis (Thorell, 1871)}

1879 Erigone vexatrix non O. Pickard-Cambridge. - L. Koch 45, T. 2, f. 2, 2a-e, $\sigma^{7} \&$ 9 , in part sensu Holm [1973].

1973 Hilaira glacialis. - Holm: 84.

1985 H. glacialis. - Eskov: 124.

REMARKS. On the Vaygach Island, the species has been recorded from the Cape Greben' (No. 26) [L. Koch, 1879; Holm 1973] and the Cape Bolvanskiy Nos (No. 24) [Eskov, 1985].

\section{Mecynargus sphagnicola (Holm, 1939)}

NEW MATERIAL EXAMINED. 1 ㅇ, Vaygach Island, Lyamchin Peninsula, environs of Cape Bol'shoy Lyamchin Nos (No. 28), bank of brook, $69.857806^{\circ} \mathrm{N} 59.147222^{\circ} \mathrm{E}, 4 \mathrm{~m}$ a.s.1., 7.VIII.2015, leg. A. Krasheninnikov, det. S.E.

REMARKS. The species is new to the fauna of the Vaygach Island.

Oreoneta leviceps (L. Koch, 1879) $\&$ ․

1879 Erigone leviceps. - L. Koch: 63, T. 2, f. 15, 15a-c, О

1973 Hilaira leviceps. - Holm: 85.

REMARKS. On the Vaygach Island, the species has been recorded from the Cape Greben'(No. 26) [L. Koch, 1879; Holm 1973].

\section{Pelecopsis parallela (Wider, 1834)}

1994 Pelecopsis parallela. - Eskov, Marusik: 75.

REMARKS. On the Vaygach Island, the species has been recorded from Talata River (No. 27) [Eskov, Marusik, 1994].
Praestigia groenlandica Holm, 1967

1994 Praestigia groenlandica. - Eskov, Marusik: 75.

2008 P. makarovae. - Marusik et al.: 221, figs 8, 16, 23, 42, 48, 54-56, 74-76, 92-93, 102-103, 106, 111, 115-116, 120, ᄋ \& ㅇ.

NEW MATERIAL EXAMINED. $1 \sigma^{7}$ subad. (PSU), Vaygach Island, Lyamchin Peninsula, environs of Cape Bol'shoy Lyamchin Nos (No. 28), middle flow of brook, on bank, $69.860027^{\circ} \mathrm{N}$ $59.133420^{\circ} \mathrm{E}, 10 \mathrm{~m}$ a.s.1., 7.VIII.2015, leg. A. Krasheninnikov, det. S.E.

REMARKS. On the Vaygach Island, the species has been recorded from Talata River (No. 27) [Eskov, Marusik, 1994]. I consider $P$. groenlandica as a circumpolar species, unlike Marusik et al. [2008] who split it into several species associated with different sectors of the Arctic. The results of further studies on $P$. groenlandica will be presented and discussed elsewhere (Tanasevitch, in preparation).

Semljicola alticola (Holm, 1950)

NEW MATERIAL EXAMINED. $18 \sigma^{\top} \sigma^{\top}, 29$ + 9 (PSU), Vaygach Island, Lyamchin Peninsula, environs of Cape Bol'shoy Lyamchin Nos (No. 28), swamp with Pedicularis, $69.856211^{\circ} \mathrm{N}$ $59.168394^{\circ} \mathrm{E}, 1 \mathrm{~m}$ a.s.1., 7.VIII.2015, leg. A. Krasheninnikov, det. S.E.; $3 \sigma^{7} \sigma^{7}, 6$ 우, same locality, middle flow of brook, on bank, $69.860027^{\circ} \mathrm{N} 59.133420^{\circ} \mathrm{E}, 10 \mathrm{~m}$ a.s.1., 7.VIII.2015, leg. A. Krasheninnikov, det. S.E.

REMARKS. The species is new to the fauna of the Vaygach Island.

Semljicola arcticus (Eskov, 1989)

1985 Latithorax sp. — Eskov: 124

1989 L. arcticus. - Eskov: 101.

REMARKS. On the Vaygach Island, the species has been recorded from the Cape Bolvanskiy Nos (No. 24) [Eskov, 1985; 1989].

\section{Semljicola barbiger (L. Koch, 1879)}

1879 Erigone barbigera. - L. Koch: 65, T. 2, f. 16, 16a, o'. 1973 Eboria barbigera. - Holm: 82, fig. 29, $\sigma^{7}$.

1994 Semljicola barbigera. - Eskov, Marusik: 59

REMARKS. On the Vaygach Island, the species has been recorded from the Cape Greben' (No. 26) [L. Koch, 1879; Holm, 1973] and Talata River (No. 27) [Eskov, Marusik, 1994].

Silometopoides sphagnicola Eskov et Marusik, 1992

NEW MATERIAL EXAMINED. 1 \% , Vaygach Island, Lyamchin Peninsula, environs of Cape Bol'shoy Lyamchin Nos (No. 28), middle flow of brook, $69.860027^{\circ} \mathrm{N} 59.133420^{\circ} \mathrm{E}, 10 \mathrm{~m}$ a.s.1., 7.VIII.2015, leg. A. Krasheninnikov, det. S.E.

REMARKS. This species is new to the fauna of the Vaygach Island.

Walckenaeria clavicornis (Emerton, 1882)

1985 Cornicularia clavicornis. - Eskov: 125.

REMARKS. On the Vaygach Island, the species has been recorded from the Cape Bolvanskiy Nos (No. 24) [Eskov, 1985]. 
ACKNOWLEDGEMENTS. I am most grateful to Vitaly M. Spitsyn, Andrei B. Krasheninnikov, Maria V. Gavrilo, Ilia N. Mordvintsev and Nikita G. Platonov whose collections have been used here. Thanks go to Sergei L. Esyunin who also supplied me with material, both identified fresh and housed in the PSU collection, to Kirill G. Mikhailov for his valuable comments on the manuscript, to Theo Blick who provided me with some old papers, as well as to Sergei I. Golovatch who kindly checked the English of an early draft of the text. This study was supported in part by the Russian Foundation for Basic Research, project No. 15-0405964 A; the Ministry of Education and Science of Russian Federation, No. 6.2343.2017; the "Arctic Floating University - 2015" International Expedition; the RGO Project "Study of the polar bear in the Russian Arctic"; the Russian-Norwegian Project SEATRACK; the Association "Maritime Heritage: Explore and Save" (St. Petersburg); as well as by the UNDP/GEF/Ministry of Natural Resources and the National Park "Russian Arctic".

\section{References}

Braendegaard J. 1958. Araneida // The Zoology of Iceland. Ejnar Munksgaard, Copenhagen. Vol.3. Part 54. S.1-113.

Crawford R.L. 1988. An annotated checklist of the spiders of Washington // Burke Museum Contributions in Anthropology and Natural History. Vol.5. P.1-48

Dahl M. 1928. Spinnen (Araneae) von Nowaja Semlja // Rep. Sci. Res. Exp. Novaja Zemlya 1921. Oslo. No.36. S.1-39.

Ehlers E. 1873a. Zur Kenntnis der Fauna von Nowaja-Semlja // Sitz. Phys. Med. Ges. Erlangen. Bd.5. S.7-10.

Ehlers E. 1873b. On the fauna of Nowaja Semlja // Ann. Mag. Nat. Hist. $4^{\text {th }}$ ser. Vol.11. No.66. P.464-465.

Eskov K.Y. 1985. [Spiders of the tundra zone of the USSR] // Ovcharenko V.I. (ed.). The fauna and ecology of spiders of the
USSR. Trudy Zool. Inst. Akad. nauk SSSR, Leningrad. Vol.139. P.121-128 [in Russian].

Eskov K.Y. 1988. The spider genera Savignya Blackwall, Diplocephalus Bertkau and Archaraeoncus Tanasevitch (Aranei, Linyphiidae) in the fauna of Siberia and the Soviet Far East // Folia Entomol. Hung. T.49. P.13-39.

Eskov K.Y. 1989. New Siberian species of erigonine spiders (Arachnida, Aranei, Linyphiidae) // Spixiana. Bd.11. H.2. S.97-109.

Eskov K.Y., Marusik Y.M. 1994. New data on the taxonomy and faunistics of North Asian linyphiid spiders (Aranei Linyphiidae) // Arthropoda Selecta. Vol.2 (for 1993). No.4. P.41-79.

Fedotov D.M. 1912. [To the spider fauna of Murman and Novaya Zemlya] // Annu. Mus. Zool. Ac. Sc. St-Petersb. Vol.16 (1911). No.4. P.449-474 [in Russian].

Heuglin M.T. 1874. Reisen nach dem Nordpolarmeer in den Jahren 1870 und 1871. III. Theil: Beiträge zur Fauna, Flora und Geologie von Spitzbergen und Novaja Semlja. Braunschweig. S.IVIII, 1-352.

Holm Å. 1973. On the spiders collected during the Swedish expedition to Novaya Zemlya and Yenisey in 1875 and 1876 // Zool. Scripta. Vol.2. No.3. P.71-110.

Koch L. 1879. Arachniden aus Sibirien und Novaja Semlja eingesammelt von der schwedischen Expedition im Jahre 1875 // Kongl. Svenska Vet.-Akad. Handl. Bd.16. H.5. S.1-136, Taf.I-VII.

Matveeva N.V. 1998. [Zonation of Arctic Vegetation] // Trudy BIN im. V.L. Komarova Ross. Akad. Nauk. St-Petersburg. Nauka Publ. 200 pp. [in Russian].

Paquin P., Buckle D.J., Dupérré N. \& Dondale C.D. 2010. Checklist of the spiders (Araneae) of Canada and Alaska // Zootaxa. Vol.2461. P.1-170.

Tanasevitch A.V. 2017. New records of spiders (Aranei) from the Russian Arctic // Arthropoda Selecta. Vol.26. No.1. P.77-82.

Tanasevitch A.V., Nekhaeva A.A. 2016. Spiders (Aranei) of the Kharaulakh Mountains and the Lena River lower reaches, northern Yakutia, Russia // Arthropoda Selecta. Vol.25. No.3. P.307326.

Responsible editor K.G. Mikhailov 